\title{
communications physics
}

ARTICLE

\section{A weakly-interacting many-body system of Rydberg polaritons based on electromagnetically induced transparency}

Bongjune Kim¹, Ko-Tang Chen', Shih-Si Hsiao${ }^{1}$, Sheng-Yang Wang ${ }^{1}$, Kai-Bo Li ${ }^{1}$, Julius Ruseckas ${ }^{2}$, Gediminas Juzeliūnas ${ }^{2}$, Teodora Kirova ${ }^{3}$, Marcis Auzinsh ${ }^{4}$, Ying-Cheng Chen ${ }^{5,6}$, Yong-Fan Chen (i) ${ }^{6,7} \&$ Ite A. Yu(D) 1,6凶

The combination of Rydberg atoms and electromagnetically induced transparency (EIT) has been extensively studied in the strong-interaction regime. Here we proposed utilizing an EIT medium with a high optical depth (OD) and a Rydberg state of low principal quantum number to create a many-body system of Rydberg polaritons in the weak-interaction regime. The phase shift and attenuation induced by the dipole-dipole interaction (DDI) were still significant, and can be viewed as the consequences of elastic and inelastic collisions among Rydberg polaritons. We further observed that the width of the transverse momentum distribution of Rydberg polaritons at the exit of the system became notably smaller as compared with that at the entrance. The observation demonstrates the cooling effect in this system. The $\mu$ s-long interaction time due to the high-OD EIT medium plus the $\mu \mathrm{m}^{2}$-size collision cross section due to the DDI suggests a feasible platform of polariton Bose-Einstein condensation.

\footnotetext{
${ }^{1}$ Department of Physics, National Tsing Hua University, Hsinchu, Taiwan. ${ }^{2}$ Institute of Theoretical Physics and Astronomy, Vilnius University, Vilnius, Lithuania. ${ }^{3}$ Institute of Atomic Physics and Spectroscopy, University of Latvia, Riga, Latvia. ${ }^{4}$ Laser Centre, University of Latvia, Riga, Latvia. ${ }^{5}$ Institute of Atomic and Molecular Sciences, Academia Sinica, Taipei, Taiwan. ${ }^{6}$ Center for Quantum Technology, Hsinchu, Taiwan. ${ }^{7}$ Department of Physics, National

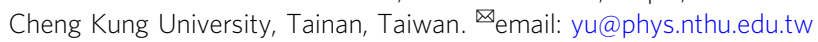


$\mathrm{R}$ ydberg-state atoms provide a strong dipole-dipole interaction (DDI) and a dipole blockade mechanism ${ }^{1-4}$ suitable for applications in quantum information processing, such as quantum simulators ${ }^{5-7}$, quantum logic gates ${ }^{8-11}$, and singlephoton sources ${ }^{12-14}$. The effect of electromagnetically induced transparency (EIT) reduces the propagation speed of light in media and significantly increases the light-matter interaction time ${ }^{15-19}$. Thus, the EIT effect involving the Rydberg atoms can efficiently mediate significant photon-photon interactions ${ }^{20-25}$, and this issue has attracted a great deal of attention recently. Most of the previous Rydberg-EIT studies concentrated on stronglycorrelated photons in the dipole blockade regime, which emerges for the strongly-interacting Rydberg atoms with a high principal quantum number, $n$, close to 100 . This leads to, for example, the two-body photon-photon gates ${ }^{11,22}$ and strongly-correlated many-body phases 21,25 .

The system of the Rydberg EIT polaritons is an ensemble of bosonic quasiparticles ${ }^{26,27}$. The dispersion relation and momentum distribution of the Rydberg polaritons can be associated with their effective mass and the temperature ${ }^{28}$. The phase shift and attenuation of the output light induced by the DDI can be viewed as a consequence of the elastic and inelastic collisions among the polaritons. Hence, the change rates of the phase shift and attenuation are related to the elastic and inelastic collision rates, respectively. Finally, the propagation time of slow light or the diffusion time of stationary light in the EIT system is exactly the interaction time of the particles.

Here we proposed and experimentally demonstrated an innovative idea of utilizing a medium with a high optical depth (OD) and Rydberg atoms with a low principal quantum number to create a weakly-interacting many-body system based on the EIT effect. In this work, $\mathrm{OD} \approx 80$ and $n=32$ were used. We studied the EIT polaritons also known as the dark-state polaritons ${ }^{26,27,29}$, which, in the case of the Rydberg EIT, are quasi-particles representing superpositions of photons and Rydberg coherences. The Rydberg coherence is the coherence between the atomic ground and Rydberg states. Weakly-interacting Rydberg polaritons under an OD-enhanced interaction time can be employed in the study of many-body physics, such as the Bose-Einstein condensation $(\mathrm{BEC})^{28,30-36}$.

The low- $n$ Rydberg state, together with a moderate polariton density, ensured the weak-interaction regime in our experiment, i.e., $r_{B}^{3} / r_{a}^{3} \ll 1$, where $r_{B}$ is the blockade radius and $r_{a}$ is the half mean distance between Rydberg polaritons. In this regime, the mean number of Rydberg polaritons in a blockade sphere is much less than the unity, and thus attenuation due to the blockade effect occurs rarely. The collision cross-section of polaritons due to the DDI is around $\mu \mathrm{m}^{2}$ in the Rydberg state of $n=32$, leading to the elastic collision rate of a few $\mathrm{MHz}$ at the Rydberg-polariton density of $2 \times 10^{9} \mathrm{~cm}^{-3}$ used in this work. On the other hand, the high-OD medium provides for the Rydberg polaritons a long interaction time of a few $\mu$ s, as demonstrated here and in our previous works ${ }^{17,18}$. Therefore, a high OD can result in a sufficiently large product of the collision rate and interaction time for weakly-interacting Rydberg polaritons, enabling them to exhibit many-body phenomena ${ }^{28,37,38}$.

In this work, we systematically studied the phase shift and attenuation of an output probe light for the EIT involving a Rydberg state of $\left|32 D_{5 / 2}\right\rangle$. Owing to the high OD and low intrinsic decoherence rate in the system, to the best of our knowledge, our experiment is the first one to demonstrate the DDI effect in the truly weak-interaction regime, i.e., $r_{B}^{3} / r_{a}^{3}<0.1$, as compared with previous experiments ${ }^{20-22,39-44}$. We were able to clearly observe the DDI-induced attenuation and phase shift even at $r_{B}^{3} / r_{a}^{3}=0.02$. Furthermore, we varied the DDI strength via the input photon flux and measured the transverse momentum distribution of the Rydberg polaritons. A larger DDI strength caused the width of the momentum distribution to become notably smaller, indicating the thermalization process was driven by elastic collisions $28,45,46$. The observed reduction of the momentum distribution width suggested that the combination of the $\mu \mathrm{m}$-range DDI strength and the $\mu \mathrm{s}$-long light-matter interaction time can make the BEC of the Rydberg polaritons feasible.

\section{Results}

Experimental setup. We carried out the experiment in cold ${ }^{87} \mathrm{Rb}$ atoms produced by a magneto-optical trap (MOT). There were typically $5 \times 10^{8}$ trapped atoms with a temperature of about $350 \mu \mathrm{K}$ in the $\mathrm{MOT}^{47,48}$. The cigar-shaped atomic cloud had a dimension of $1.8 \times 1.8 \times 6.0 \mathrm{~mm}^{3}$. Before each measurement, we first turned off the magnetic field of the MOT and then performed the dark MOT for $2.5 \mathrm{~ms}$ to increase the OD of the system $^{18,49}$. The OD of the atomic cloud was $81 \pm 3$. When the experimental condition of a low OD was needed, we were able to adjust the dark-MOT parameter to reduce the OD of the system by about fourfolds.

Figure 1a shows the transitions driven by the probe and the coupling fields in the EIT system. We optically pumped all population to a single Zeeman state $\left|F=2, m_{F}=2\right\rangle$ at the ground level $\left|5 S_{1 / 2}\right\rangle{ }^{49}$. Since the probe and coupling fields were $\sigma_{+}$-polarized, each of the energy levels $|1\rangle,|2\rangle$ and $|3\rangle$ shown in Fig. 1a was a single Zeeman state. The spontaneous decay rate, $\Gamma$, of $|3\rangle$ is $2 \pi \times 6 \mathrm{MHz}$, and $|1\rangle \leftrightarrow|3\rangle$ is a cycling transition. The lifetime, $\Gamma_{2}^{-1}$, of $|2\rangle$ is about $30 \mu s^{50}$, and $\Gamma_{2} \ll \gamma_{0}$ in this work, where $\gamma_{0}$ is the intrinsic decoherence rate of the system. Thus, the influence of $\Gamma_{2}$ was negligible. We estimated $n_{\text {atom }} \approx 5 \times 10^{10} \mathrm{~cm}^{-3}$ in the experiment, and $C_{6}=-2 \pi \times 260 \mathrm{MHz} \cdot \mu \mathrm{m}^{6}$ for $\left|32 D_{5 / 2}, m_{J}=5 / 2\right\rangle$ by using $D_{\varphi}=0.343$, where $D_{\varphi}$ is the coefficient resulted from the angular momentum states of two interacting Rydberg atoms being $\left|J=5 / 2, m_{J}=5 / 2\right\rangle^{51}$. Such $C_{6}$ together with $n_{\text {atom }}$ can make the DDI effect unobservable under a small $\Omega_{p}$ or a low $\mathrm{OD}^{20,39,52,53}$.

Figure $1 \mathrm{~b}$ shows the experimental setup. The probe field came from the first-order beam of an acousto-optic modulator (AOM)

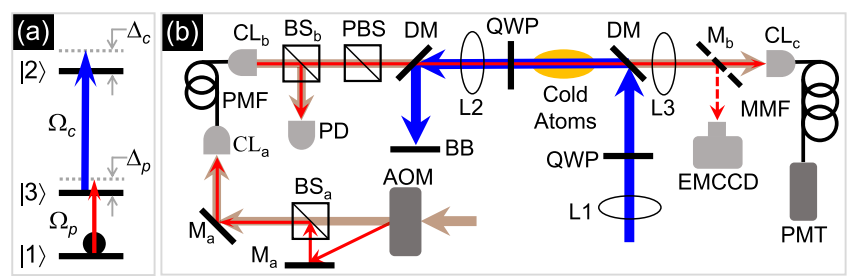

Fig. 1 Transition scheme and experimental setup. a Relevant energy levels and laser excitations in the experiment. States $|1\rangle,|2\rangle$, and $|3\rangle$ correspond to the ground state $\left|5 S_{1 / 2}, F=2, m_{F}=2\right\rangle$, Rydberg state $\left|32 D_{5 / 2}, m_{J}=5 / 2\right\rangle$, and excited state $\left|5 P_{3 / 2}, F=3, m_{F}=3\right\rangle$ of ${ }^{87} \mathrm{Rb}$ atoms. $\Omega_{c}$ and $\Omega_{p}$ denote the coupling and probe Rabi frequencies, and $\Delta_{c}$ and $\Delta_{p}$ represent their detunings. $\mathbf{b}$ Experimental setup. AOM acousto-optic modulator, $\mathrm{BS}_{a}, \mathrm{BS}_{b}$ : beam splitters, $\mathrm{CL}_{a}, \mathrm{CL}_{b}, \mathrm{CL}_{c}$ : collimation lenses, $\mathrm{PMF}$ polarization-

maintained optical fiber, $B B$ beam block, $M_{a}$ : mirror, $M_{b}$ : movable mirror on a flip mount, PD photodetector (Thorlabs APD110A), PBS polarizing beam splitter, DM dichroic mirror, L1, L2, L3: lenses, QWP quarter-wave plate, EMCCD electron-multiplying charge-coupled device camera (Andor DL604M-OEM), MMF multimode optical fiber, PMT photomultiplier tube and amplifier (Hamamatsu H6780-20 and C9663). Blue, red, and brown arrowed lines indicate the optical paths of the coupling, probe, and AOM's zeroth-order beams, respectively. 
and was coupled to a polarization maintained fiber (PMF). The PMF delivered the probe field to the cold atoms. The probe field was twice diffracted from the AOM in the double-pass scheme. The AOM was used to shape the probe pulse, and its driving rf frequency and amplitude were precisely tuned or varied by a function generator. We used a rubidium atomic standard (SRS FS752) as the external clock of the function generator. The blue path in Fig. $1 \mathrm{~b}$ represents the coupling field, which was switched on and off with another AOM not drawn in the figure. Inside the atom cloud, the probe and coupling fields counter-propagated and completely overlapped to minimize the Doppler effect. The $e^{-1}$ full widths of the probe and coupling beams were 130 and $250 \mu \mathrm{m}$, respectively. After passing through the atoms, the probe field was detected by a photomultiplier tube (PMT). A digital oscilloscope (Agilent MSO6014A) acquired the signal from the PMT and produced raw data.

The entire setup of red and brown optical paths shown in Fig. $1 \mathrm{~b}$ formed the beat-note interferometer, which measured the phase shift of the output probe field (see beat-note interferometer in "Method"). The brown optical paths were present only in the phase shift measurement.

Theoretical model. The mean-field model developed in Ref. ${ }^{4}$, which describes the DDI-induced attenuation and phase shift in the system of weakly-interacting Rydberg polaritons, is summarized in this section. Rydberg polaritons are randomly distributed and can be considered approximately as particles of a nearly ideal gas due to their weak interaction. Thus, the nearestneighbor distribution ${ }^{55}$ was utilized in our theory. Using the probability function of the nearest-neighbor distribution and the atom-light coupling equations of an EIT system, we derived the following analytical formulas of the steady-state DDI-induced attenuation coefficient $\Delta \beta$ and phase shift $\Delta \phi$ :

$$
\begin{gathered}
\Delta \beta=2 S_{\mathrm{DDI}}\left(\frac{\sqrt{W_{c}-2 \Delta_{c}}}{W_{c}}-\frac{3 \gamma_{0} \sqrt{W_{c}+2 \Delta_{c}}}{\Omega_{c}^{2}}\right) \Omega_{p}^{2}, \\
\Delta \phi=S_{\mathrm{DDI}}\left(\frac{\sqrt{W_{c}+2 \Delta_{c}}}{W_{c}}-\frac{3 \gamma_{0} \sqrt{W_{c}-2 \Delta_{c}}}{\Omega_{c}^{2}}\right) \Omega_{p}^{2}, \\
S_{\mathrm{DDI}} \equiv \frac{\pi^{2} \alpha \Gamma \sqrt{\left|C_{6}\right|} n_{\text {atom }} \varepsilon}{3 \Omega_{c}^{3}}, \\
W_{c} \equiv \sqrt{\Gamma^{2}+4 \Delta_{c}^{2}},
\end{gathered}
$$

where $\alpha$ is the OD of the system, $\Gamma$ is the spontaneous decay rate of the excited state $|3\rangle, \gamma_{0}$ is the intrinsic decoherence rate in the system, $C_{6}$ is the van der Waals coefficient in SI units of $\mathrm{Hz} \cdot \mathrm{m}^{6}$, $n_{\text {atom }}$ is the atomic density, and $\varepsilon$ is a phenomenological parameter. In the above equations, we assume $\Omega_{p}^{2} \ll \Omega_{c}^{2}$ and $r_{B}^{3} \ll r_{a}^{3}$, and consider the case that the two-photon detuning $\delta$ is much smaller than the EIT linewidth and its influence on $\Delta \beta$ and $\Delta \phi$ is negligible. Furthermore, $\varepsilon$ is utilized to relate the average Rydberg-state population $\left(\rho_{22}\right)$ to the input probe and coupling Rabi frequencies $\left(\Omega_{p}\right.$ and $\left.\Omega_{c}\right)$ as $\rho_{22}=\varepsilon \Omega_{p}^{2} / \Omega_{c}^{2}$. Equations (1) and (2) are for the case of $C_{6}<0$. They qualitatively agree with those in the pioneering work of Ref. ${ }^{56}$ and served as the theoretical references for the experimental data in this work.

The attenuation coefficient $\beta$ and phase shift $\phi$ of the probe field is defined by the output-input $\left(\Omega_{p}^{\prime}-\Omega_{p}\right)$ relation of $\Omega_{p}^{\prime}=\Omega_{p} \exp (i \phi-\beta / 2)$. Thus,

$$
\beta=\beta_{0}+\Delta \beta \approx \frac{2 \alpha \gamma_{0} \Gamma}{\Omega_{c}^{2}}+\Delta \beta,
$$
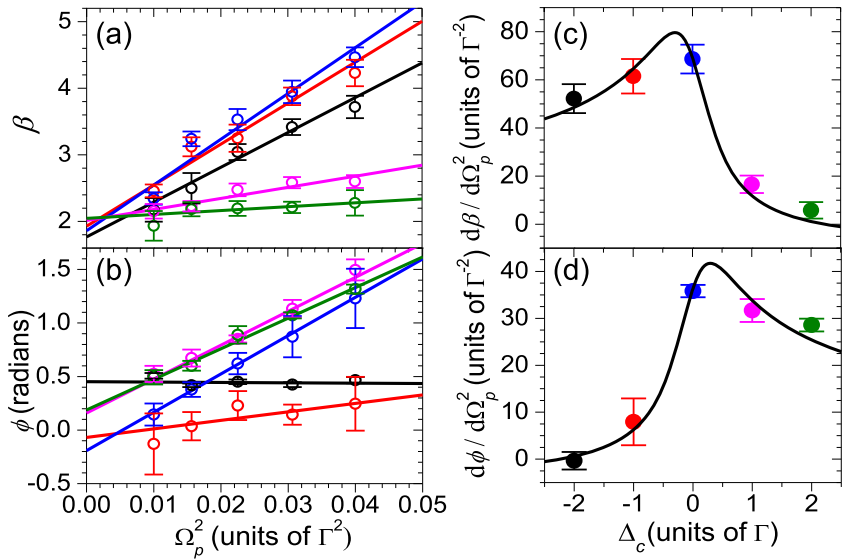

Fig. 2 Attenuation coefficient and phase shift due to the dipole-dipole interaction (DDI). a, b Attenuation coefficient $\beta$ and phase shift $\phi$ at $\delta=0$ as functions of $\Omega_{p}^{2}$ in the presence of the DDI. Circles are the experimental data taken with $\alpha$ (optical depth) $=81 \pm 3, \Omega_{c}=1.0 \Gamma$, and $\Delta_{c}=-2 \Gamma$ (black), $-1 \Gamma$ (red), 0 (blue), $1 \Gamma$ (magenta), and $2 \Gamma$ (olive), where $\Gamma=2 \pi \times 6 \mathrm{MHz}$. Straight lines are the best fits. c, $\mathbf{d}$ Circles are data points of the slope of a straight line in (a) and (b) versus the corresponding $\Delta_{c}$. Black lines are the best fits, which determine $S_{D D I}=37 / \Gamma^{3 / 2}$ in (c) and $38 / \Gamma^{3 / 2}$ in (d). The error bars represent the standard deviation of measured values.

$$
\phi=\phi_{0}+\Delta \phi \approx \frac{\alpha \Gamma \delta}{\Omega_{c}^{2}}+\Delta \phi,
$$

where $\beta_{0}$ and $\phi_{0}$ are the attenuation coefficient and phase shift in the absence of $\mathrm{DDI}^{54}, \delta\left(=\Delta_{p}+\Delta_{c}\right)$ is the two-photon detuning. The mean-field theory predicted that both $\beta$ and $\phi$ depend on $\Omega_{p}^{2}$ linearly, and the dependence of $\Omega_{p}^{2}$ comes from the Rydberg-state population $\rho_{22}$. In addition, the slope of $\beta$ or $\phi$ versus $\Omega_{p}^{2}$ is asymmetric with respect to $\Delta_{c}=0$.

DDI-induced attenuation and phase shift. We first verified that the DDI effect can be observed in the system consisting of weakly-interacting Rydberg polaritons. The attenuation coefficient $\beta$ and phase shift $\phi$ were measured as functions of the square of the probe Rabi frequency $\Omega_{p}^{2}$, as shown in Fig. 2a, b. A nonzero two-photon detuning, $\delta$, can significantly affect the phase shift and attenuation of the output probe light in the EIT medium. Thus, we utilized a beat-note interferometer to carefully determine the probe frequency for $\delta=0$ (see beat-note interferometer in "Method"). The uncertainty of $\delta / 2 \pi$ was $\pm 30 \mathrm{kHz}$, including the accuracy of the beat-note interferometer of $\pm 10 \mathrm{kHz}$ and the long-term drift of the two-photon frequency of $\pm 20 \mathrm{kHz}$.

At $\delta=0$, we applied a square input probe pulse and measured the steady-state attenuation coefficient $\beta$ and phase shift $\phi$ of the output probe pulse. The measurement procedure and representative data can be found in Supplementary Note 3. Figure 2a (and b) shows $\beta$ (and $\phi$ ) versus $\Omega_{p}^{2}$ at various $\Delta_{c}$, where $\Omega_{p}^{2}$ corresponds to the peak or center intensity of the input Gaussian beam. At a given $\Delta_{c}$, the data points approximately formed a straight line, as expected from the theory. We fitted the data with a linear function and plotted the slope of the best fit against $\Delta_{c}$, as shown in Fig. 2c, d. It can be noticed that the $y$-axis interceptions of best fits were scattered in Fig. 2b. Since a change of $30 \mathrm{kHz}$ in $\delta / 2 \pi$ resulted in that of $0.4 \mathrm{rad}$ in phase, the uncertainty of $\delta$ made the interceptions scatter around zero.

The strengths of the DDI effect on $\beta$ and $\phi$, i.e., the slope of $\beta$ versus $\Omega_{p}^{2}$ and that of $\phi$ versus $\Omega_{p}^{2}$ (denoted as $\chi_{\beta}$ and $\chi_{\phi}$ ), were 
asymmetric with respect to $\Delta_{c}=0$ as shown in Fig. $2 \mathrm{c}$, d. Such asymmetries are expected from the theory, as demonstrated by Eqs. (1) and (2). Considering the coupling detunings of $-\left|\Delta_{c}\right|$ and $\left|\Delta_{c}\right|, \chi_{\beta}$ (or $\chi_{\phi}$ ) of $-\left|\Delta_{c}\right|$ was always larger (or smaller) than that of $\left|\Delta_{c}\right|$. The physical picture of the asymmetries is discussed in Ref. ${ }^{54}$. We fitted the data points in Fig. $2 c$, $d$ with the functions of $\chi_{\beta}$ and $\chi_{\phi}$, where $\gamma_{0}$ was set to $0.012 \Gamma$, and $S_{\text {DDI }}$ was the only fitting parameter. The $S_{\mathrm{DDI}}$ of the best fit shown in Fig. $2 \mathrm{c}$ is in good agreement with that of Fig. 2d. Using Eq. (3) and the values of $S_{\mathrm{DDI}}, C_{6}, n_{\text {atom }}$, and $\Omega_{c}$, we obtained $\varepsilon=0.43 \pm 0.05$. Considering that $\varepsilon$ related the average Rydberg-state population to $\Omega_{p}^{2}$ and that $\Omega_{p}^{2}$ corresponded to the center intensity of the input Gaussian beam, the measured value of $S_{\mathrm{DDI}}$ is also reasonable. Thus, the experimental data are in good agreement with the theoretical predictions, revealing that the low- $n$ Rydberg polaritons in the high-OD medium can form a weakly-interacting many-body system.

Feasibility of thermalization process. To observe the thermalization process in this weakly-interacting system, we varied the DDI strength and measured the transverse momentum distribution of the Rydberg polaritons. The moveable mirror $\mathrm{M}_{b}$ has shown in Fig. 1b was installed to direct the output probe beam to the EMCCD. Figure 3 shows the key elements for the EMCCD image of the probe beam. We reduced the waist of the input probe beam by adding the lens pair of L4 and L5, as shown in the figure, and made the input photons or initial Rydberg polaritons have a large transverse momentum distribution. In the measurements of Figs. $4-7$, the $\mathrm{e}^{-1}$ full width of the probe beam at the center of the atomic cloud was reduced to $39 \mu \mathrm{m}$, while we kept the coupling beam size the same as before. Since the coupling beam size was 6.4 times larger than the probe beam size, the waveguide effect due to the coupling intensity profile should play little role in the data of these figures. Furthermore, the attenuation of Rydberg polaritons decreases the particle density and consequently the collision rate. While the propagation delay time, i.e., the interaction time, at $\Delta_{c}$ of a small magnitude can be maintained approximately the same, the DDI-induced attenuation coefficient of $\Delta_{c}=+1 \Gamma$ is significantly smaller than those of $\Delta_{c}=0$ and $-1 \Gamma$. Hence, we chose the experimental condition of $\Delta_{c}=+1 \Gamma$ in the study.

Following the idea in Ref. ${ }^{28}$, we estimate the elastic collision rate, which is responsible for the thermalization, of the Rydberg polaritons from the formula below:

$$
\frac{d \phi}{d t}=R_{c} \phi_{c}
$$

where $d \phi / d t$ is the phase shift per unit time, $R_{c}$ denotes the collision rate, and $\phi_{c}$ represents the phase shift per collision. Note that in the case of $\Delta_{c}=-1 \Gamma$, for example, some collisions result in positive phase shifts and some result in negative phase shifts ${ }^{54}$, and thus the net phase shift is close to zero. Such a case cannot be used to make an estimation of the collision rate. To ensure that the phase shifts induced by the collisions are all positive, we should use the case of $\Delta_{c}=0$ or $+1 \Gamma$ in the estimation. The value of $d \phi / d t$ can be estimated from the experimentally observed value of $\Delta \phi / \tau_{d}$, where $\Delta \phi$ is the DDI-induced phase shift measured at the output of the medium and $\tau_{d}$ is the propagation delay time, i.e., the interaction time of Rydberg polaritons. Using $\tau_{d}=2.1 \mu \mathrm{s}$ and the data point is shown in Fig. $2 \mathrm{~b}$ of $\Delta_{c}=+1 \Gamma$ and $\Omega_{p}=0.2 \Gamma$ resulted in $d \phi / d t=0.64 \mathrm{rad} / \mu \mathrm{s}$. Note that the experimental condition of $\Omega_{p}=0.2 \Gamma$ corresponded to the Rydberg-polariton density of $2 \times 10^{9} \mathrm{~cm}^{-3}$. Considering the hard-sphere collision, $\phi_{c}=\bar{k} a$ where $\hbar \bar{k}$ is the root-mean-square value of the relative momentum between two colliding bodies, and $a$ is the radius of a

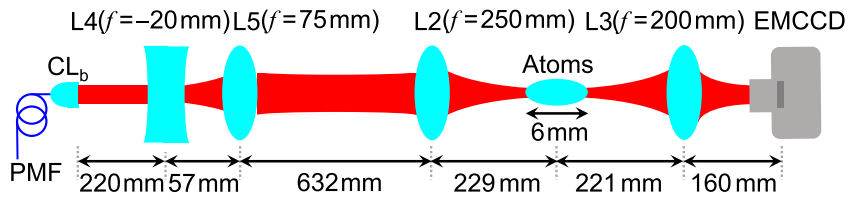

Fig. 3 Sketch of key elements for taking images of the probe beam. Values of $f$ indicate the focal lengths of lenses. PMF polarization-maintained optical fiber; $\mathrm{CL}_{b}$ : collimation lenses, $\mathrm{L} 2$ and $\mathrm{L} 3$ : lenses, $\mathrm{EMCCD}$ electronmultiplying charge-coupled device camera (Andor DL-604M-OEM). The probe beam coming out of $\mathrm{CL}_{b}$ was collimated and had the $e^{-1}$ full width of $0.92 \mathrm{~mm}$. We adjusted the separation between L4 and L5 such that the beam was focused on nearly the center of the atomic cloud by $L 2$, and had the width of $39 \mu \mathrm{m}$ at the focal point.

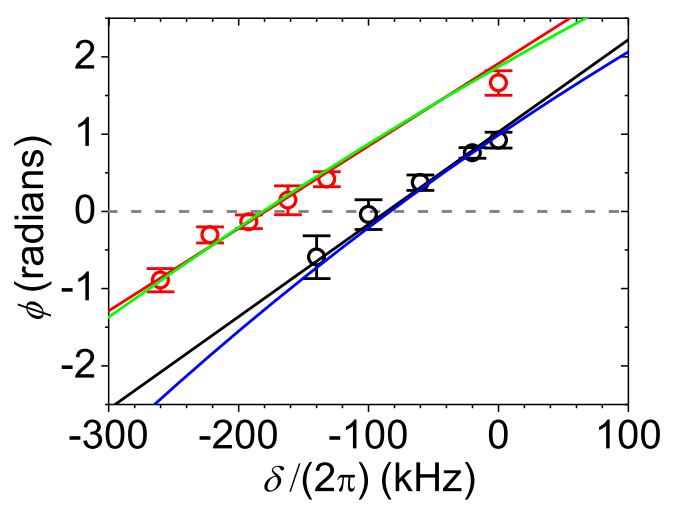

Fig. 4 Determination of the two-photon detuning for the zero phase shift. Black (or red) circles are the experimental data of phase shift versus twophoton detuning measured at $\alpha$ (optical depth) $=82, \Omega_{c}=1.0 \Gamma, \Delta_{c}=1.0 \Gamma$, and $\Omega_{p}=0.10 \Gamma$ (or $0.20 \Gamma$ ). Black and red lines represent the linear best fits. We determined the two-photon detuning, $\delta_{0}$, for the zero phase shift, i.e., $\phi=0$, by the intersection between the best fit and the gray dashed line. As references, blue and green lines are the theoretical predictions calculated numerically ${ }^{54}$. The error bars represent the standard deviation of measured values.

hard-sphere. In view of the Rydberg polaritons, $a$ can be treated as the blockade radius $^{57}$, which was about $2.1 \mu \mathrm{m}$ in our case according to the formula ${ }^{21}$ of $r_{B}=\left(2 C_{6} \Gamma / \Omega_{c}^{2}\right)^{1 / 6}$. The momentum distribution, which we measured in this study and will be shown later, indicated $\bar{k}=0.051 \mu \mathrm{m}^{-1}$. The values of $\bar{k}$ and $a$ result in $\phi_{c}=0.11 \mathrm{rad}$. Thus, we obtain $R_{c}=6.0 \mathrm{MHz}$ by inserting the values of $d \phi / d t$ and $\phi_{c}$ in Eq. (7). Under such an elastic collision rate, it was feasible to observe the thermalization effect in our experiment.

Procedure of the beam profile measurement. We measured the output probe beam size on the EMCCD at $\Delta_{c}=1 \Gamma$. To avoid the lensing effect ${ }^{58}$, we set the two-photon detuning, $\delta_{0}$, corresponding to the zero phase shift, giving $\phi=0$ in the measurement. That is, at the two-photon resonance $(\delta=0)$ there is a positive DDI-induced phase shift $(\phi>0)$, and we deliberately set a negative two-photon detuning, $\delta_{0}$, to eliminate the phase shift, i.e., $\phi=0$ at $\delta=\delta_{0}$ where $\delta_{0} / 2 \pi$ was equal to -85 or $-180 \mathrm{kHz}$ in the measurement of $\Omega_{p}=0.1 \Gamma$ or $0.2 \Gamma$, respectively. Please note $\phi$ $=0$ is the condition that the phase shift due to the ordinary EIT effect at $\delta_{0}$ cancels out the phase shift due to the DDI effect. Details of the measurement of the probe beam size, i.e., the transverse momentum distribution of the Rydberg polaritons, and those of the study on the lensing effect can be found in Supplementary Notes 4 and 5 . 
The procedure of taking the image of the output probe beam profile is summarized in the following steps: (i) the experimental parameters were determined and set to the designed values. (ii) The probe frequency for $\delta=0$ was determined by the method of the beat-note interferometer. (iii) At the above experimental parameters, we measured $S_{\mathrm{DDI}}$, and confirmed that the measured value was consistent with the $S_{\mathrm{DDI}}$ shown in Fig. 2 . (iv) The twophoton detuning, $\delta_{0}$, corresponding to $\phi=0$ was determined. Representation data are shown in Fig. 4. We fitted the data with a straight line, and the best fit determined $\delta_{0}$. The uncertainty of $\delta_{0}$ was $\pm 2 \pi \times 30 \mathrm{kHz}$. (v) At $\delta_{0}$, we took images of the output probe beam profile. We repeated the steps (iv) and (v) for different values of $\Omega_{p}$. (vi) The value of $S_{\text {DDI }}$ was measured again to verify it was unchanged during the steps (iv) and (v).

Images of the probe beam profile. Figure $5 \mathrm{a}$ shows the probe beam profile in the absence of the atom, while Fig. 5b, c show those in the presence of the atoms. It can be clearly observed that a larger value of $\Omega_{p}^{2}$, i.e., a higher density of Rydberg polaritons because of $n_{\text {atom }} \rho_{22} \propto \Omega_{p}^{2}$, caused the beam size to become smaller. Since transverse momentums of the Rydberg polaritons were carried by the probe photons leaving the medium, the intensity profile of the output probe beam was able to be used to derive the transverse momentum distribution ${ }^{28,33}$. We fitted the intensity profiles of three images with a two-dimension Gaussian function and utilized the results of the best fits to construct the momentum distributions shown in Fig. 5d-f. The relation

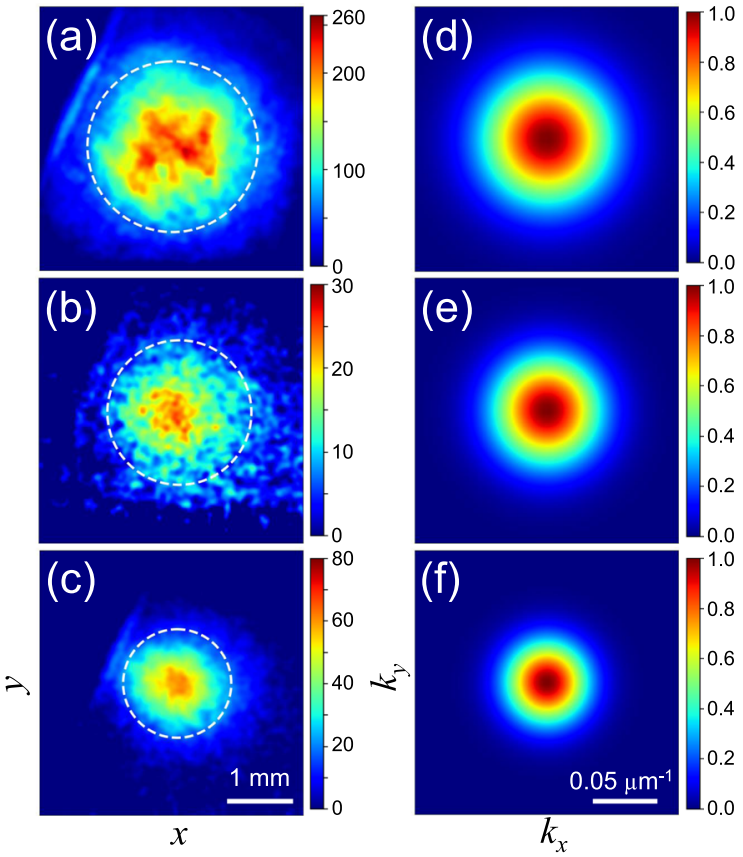

Fig. 5 Images of the output probe beam profile and transverse momentum distributions of the Rydberg polaritons. a-c Images of the probe beam profile taken by the electron-multiplying charge-coupled device camera (EMCCD) at $\Omega_{p}=0.2 \Gamma$ in the absence of the atoms, and at $\Omega_{p}=$ $0.1 \Gamma$ and $0.2 \Gamma$ in the presence of the atoms, respectively. Color represents the gray level detected by the EMCCD. We fit each image with a Gaussian function and draw a white-dashed-line circle of the diameter equal to the $e$

${ }^{-1}$ full width of the best fit. The diameters of the circles from top to bottom are 2.6, 2.2, and $1.6 \mathrm{~mm}$. d-f Transverse momentum distributions of the probe photons at the output, i.e., the Rydberg polaritons in the atomic cloud, derived from the best fits of the images in (a)-(c), respectively. Color represents the normalized probability density. The $e^{-1}$ full widths of the distributions from top to bottom are $0.10,0.087$, and $0.065 \mu \mathrm{m}^{-1}$. between the transverse momentum distribution of photons at the atom cloud and the intensity profile of an EMCCD image can be found in Supplementary Note 6. As the Rydberg-polariton density increased, the elastic collision rate also increased. Due to $\Omega_{c}$ $=1.0 \Gamma$, the values of $\Omega_{p}$ in the cases of Fig. $5 b, c$ well satisfied the perturbation condition. Thus, the propagation times of the probe light, i.e., the interaction times of the Rydberg polaritons, were approximately the same in the two cases. Under the same interaction time, the higher collision rate due to the larger Rydbergpolariton density produced a smaller width of the transverse momentum distribution or a lower effective transverse temperature, which is the expected outcome of the thermalization process.

To check whether the uncertainty of $\delta_{0}$ could be significant on the probe beam profile due to the lensing effect, we measured the probe beam size not only at $\delta_{0}$ but also at $\delta_{0} \pm 2 \pi \times 50 \mathrm{kHz}$. Figure 6 shows the measured beamwidths at these two-photon detunings. As $\Omega_{p}=0.1 \Gamma, \delta_{0}=-2 \pi \times 85 \mathrm{kHz}$. As $\Omega_{p}=0.2 \Gamma, \delta_{0}=$ $-2 \pi \times 180 \mathrm{kHz}$. Both values of $\delta_{0}$ were determined in the way illustrated by Fig. 4. In Fig. 6, the gray dashed line is the beamwidth measured without the presence of the atoms, and the black and red solid lines are the linear best fits of the experimental data. It is verified by the gentle slopes of the best fits that the uncertainty in $\delta_{0}$ and the lensing effect played insignificant roles in the measurement of the probe beamwidth.

To verify that the observed reduction of the beam size on the EMCCD image is not caused by the nonlinear self-focusing effect of the probe beam, we also measured the probe beam size at $\Delta_{c}=$ $-1 \Gamma$. As demonstrated by Fig. 2 d, the DDI-induced phase shift at $\Delta_{c}=-1 \Gamma$ is $5-6$ times smaller than that of at $\Delta_{c}=+1 \Gamma$. Consequently, the nonlinear self-focusing effect at $\Delta_{c}=-1 \Gamma$ is little as compared with that at $\Delta_{c}=+1 \Gamma$. Figure 7 shows the EMCCD images taken at $\Delta_{c}=-1 \Gamma$ with the same procedure and method as those in Fig. 5. The reduction of the probe beam size is still observed at $\Omega_{p}=0.1 \Gamma$ (or $0.2 \Gamma$ ) by comparing Fig. $7 \mathrm{a}, \mathrm{b}$ (or c) without and with the presence of atoms, respectively. Due to the large attenuation coefficient at $\Delta_{c}=-1 \Gamma$, the degree of the reduction in Fig. $7 \mathrm{c}$ is not as significant as that in Fig. $5 \mathrm{c}$, but they are comparable. Thus, the reduction of the probe beam width cannot be explained by the nonlinear self-focusing effect, and it is the consequence of the transverse momentum distribution of the Rydberg polaritons being narrowed the thermalization process.

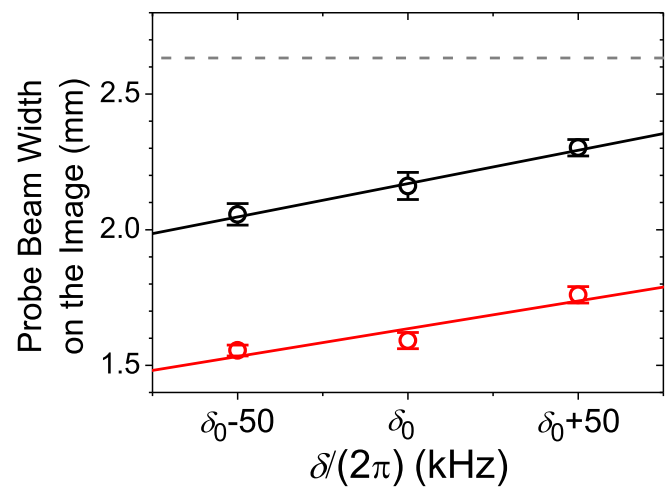

Fig. 6 The output probe beam width as a function of the two-photon detuning. Black and red circles are the experimental data of the $e^{-1}$ full width of the output probe beam versus the two-photon detuning. The black (or red) data points were taken at $\alpha$ (optical depth) $=82, \Omega_{c}=1.0 \Gamma, \Delta_{c}=$ $1.0 \Gamma$, and $\Omega_{p}=0.10 \Gamma$ (or $0.20 \Gamma$ ). Black and red lines are the linear best fits of the experimental data, and their slopes are $2.5 \mu \mathrm{m} / \mathrm{kHz}$ and $2.1 \mu \mathrm{m} / \mathrm{kHz}$, respectively. The Gray dashed line indicates the probe beamwidth measured without the presence of the atoms, i.e., the input probe beamwidth. The error bars represent the standard deviation of measured values. 

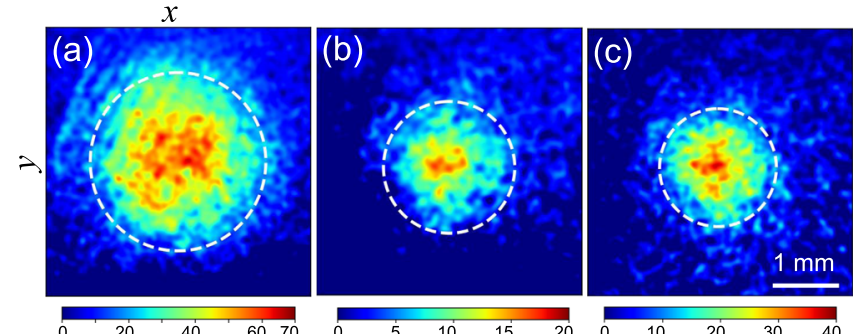

Fig. 7 Images of the output probe beam profile taken at $\boldsymbol{\Delta}_{\boldsymbol{c}}=-\mathbf{1 \Gamma}$. Here, the phase shift induced by the dipole-dipole interaction is minimal. a The image was taken in the absence of the atoms and at $\Omega_{p}=0.1 \Gamma$. b, c The images were taken in the presence of the atoms, and $\Omega_{p}=0.1 \Gamma$ and $0.2 \Gamma$, respectively. Color represents the gray level detected by the electronmultiplying charge-coupled device camera. We fit each image with a Gaussian function and draw a white-dashed-line circle of the diameter equal to the $e^{-1}$ full width of the best fit. The diameters of the circles in (a), (b), and (c) are 2.6, 2.0, and $1.8 \mathrm{~mm}$, respectively.

It is noteworthy that we did not observe any asymmetry in the images of Fig. 5b, c. In Ref. ${ }^{59}$, two Rydberg atoms were trapped in two microscopic dipole traps. The angle between the interatomic axis and the propagation direction of light fields was well defined. Thus, an anisotropic interaction as a function of the angle due to the Rydberg $D$ state was observed. In this work, the Rydberg atoms were randomly distributed. Angles between the interatomic axes and the propagation direction of light fields were also randomly distributed. Furthermore, we took the probe beam images or Rydberg-polariton momentum distributions in the plane transverse to the propagation direction of light fields or the quantization axis. As expected, the images of Fig. 5b, $c$ do not reveal any anisotropic interaction of the Rydberg $D$ state.

\section{Discussion}

Let us estimate the effective transverse temperature from Fig. 5 to show the thermalization efficiency. Following the idea in Ref. ${ }^{28}$, the effective mass of Rydberg polaritons in the transverse direction is $m_{\perp}=\eta \hbar k_{p} \Gamma / \Omega_{c}^{2}$, where $\eta$ is the OD per unit length and $k_{p}$ is the wave vector of the probe light. Suppose the Rydberg polaritons obey the Maxwell-Boltzmann distribution. Then, the effective temperature in the transverse direction is given by

$$
T_{\text {eff } \perp}=\hbar^{2} \Delta_{k}^{2} /\left(2 m_{\perp} k_{B}\right),
$$

where $\Delta_{k}$ is the $e^{-1}$ half-width of the momentum distribution and $k_{B}$ is the Boltzmann constant. Using the above formulas, we estimate the values of $T_{\text {eff } \perp}$ corresponding to Fig. $5 \mathrm{~d}$, $\mathrm{f}$ (or equivalently Fig. $5 \mathrm{a}, \mathrm{c}$ ) to be about 3.1 and $1.2 \mu \mathrm{K}$, respectively. Thus the effective temperature $T_{\text {eff } \perp}$ experiences a 2.6-fold reduction, as the Rydberg polaritons with the initial density of $2 \times 10^{9} \mathrm{~cm}^{-3}\left(\Omega_{p}=0.2 \Gamma\right)$ traverse the medium. Furthermore, Fig. $5 \mathrm{e}$ (or b) for $\Omega_{p}=0.1 \Gamma$ indicates $T_{\text {eff } \perp}=2.0 \mu \mathrm{K}$. As $T_{\text {eff } \perp}$ of Fig. $5 \mathrm{f}$ is compared with that of Fig. $5 \mathrm{e}$, a larger $\Omega_{p}$ corresponding to a higher polariton collision rate results in a better cooling effect.

In addition to the elastic collision, the observed cooling effect can be assisted by the EIT bandwidth. After an elastic collision between two Rydberg polaritons, one polariton can increase the momentum and the other can decrease the momentum. The one with a larger momentum has a higher frequency of two-photon detuning and is more likely dissipated in the medium due to the EIT bandwidth, while the other with a smaller momentum has a lower frequency and can survive well. After many elastic collisions, the average energy or temperature of the Rydberg-polariton system becomes reduced.
The authors of Ref. ${ }^{28}$ proposed to utilize stationary dark-state polaritons, i.e., stationary light based on the $\Lambda$-type EIT, to form the Bose-Einstein condensation (BEC). Please note that the interaction between such dark-state polaritons is induced by a far-detuned coupling scheme, e.g., the detuning used in Ref. ${ }^{28}$ is 50 times exceeding the spontaneous decay rate. Thus, the scattering cross-section of dark-state polaritons is far smaller than that of Rydberg polaritons interacting due to the strong interaction between the Rydberg atoms. Once Rydberg polaritons are made stationary, the BEC formulism in Ref. ${ }^{28}$ can be readily applied to the Rydberg-polariton system. The critical temperature $T_{\mathrm{c}}$ for the polariton BEC under the cylindrical symmetry is given by $^{28}$

$$
\begin{gathered}
T_{c}=\frac{\pi \hbar^{2} \sqrt[3]{n_{p}^{2}}}{k_{B} \sqrt[3]{m_{\perp}^{2} m_{\|}}} \\
m_{\perp}=\frac{\hbar \eta k_{p} \Gamma}{\Omega_{c}^{2}} \\
m_{\|}=\frac{\hbar \eta^{2} \Gamma^{2}}{8 \Omega_{c}^{2} \Delta_{c}}
\end{gathered}
$$

where $n_{p}$ is the Rydberg-polariton density, $m_{\perp}$ and $m_{\|}$are the transverse and longitudinal effective masses, $\eta$ is the OD per unit length, and $k_{p}$ is the wave vector of the probe light. However, in the present study, we dealt with a two-dimensional Rydbergpolariton system. The Rydberg polaritons propagated along the longitudinal direction. The average longitudinal kinetic energy of each Rydberg polariton ( $=\hbar k_{p} v_{g} / k_{B}$ where $v_{g}$ is the group velocity) given by $140 \mathrm{mK}$ was much larger than the transverse temperature. To achieve BEC, we need to make Rydberg polaritons stationary and further enhance OD to have a sufficient interaction time, i.e., a lifetime of the stationary light ${ }^{17}$. It is also desirable to add an artificial trap to enable efficient evaporative cooling or energy dissipation. Using $\eta=160 \mathrm{~cm}^{-1}, \Omega_{c}=1.0 \Gamma$, and $\Delta_{c}=$ $1.0 \Gamma$, we estimate $T_{\mathrm{c}}=2.0 \mathrm{mK}$ for the stationary Rydberg polaritons of $n_{p}=2 \times 10^{9} \mathrm{~cm}^{-3}$.

Up to now, the BECs of various kinds of polaritons have been realized in cavity systems ${ }^{33-36}$. This work deals with the cavityfree high-OD medium, in which the interaction time between the Rydberg polaritons is analogous to the storage time of polaritons in a cavity with a $Q$ factor greater than $10^{9}$. While the current systems of polariton BECs are all two-dimensional, a threedimension BEC can be formed in the system of Rydberg or darkstate polaritons ${ }^{28}$. In addition, as compared with other kinds of polaritons, the condensate of Rydberg polaritons can have a much longer lifetime due to a low decoherence rate in the Rydberg-EIT system, offering more opportunities for many-body physics of polariton systems.

\section{Method}

Laser fields preparation. The probe and coupling fields were generated by a homemade diode laser and a blue laser system (Toptica TA-SHG pro), respectively. The frequency stabilizations of the probe and coupling lasers are described as follows. We utilized the injection lock scheme to stabilize the frequency of the probe laser. In the scheme, the probe laser was used as the slave, and an externalcavity diode laser (ECDL) of Toptica DLC DL pro with a wavelength of about 780 nm was employed as the master. We utilized the Pound-Drever-Hall scheme and the saturated absorption spectroscopy to lock the frequency of the ECDL with a heated vapor cell of $\mathrm{Rb}$ atoms. The blue laser had a wavelength of about $482 \mathrm{~nm}$. Furthermore, we used the Pound-Drever-Hall scheme and the EIT spectrum to lock the frequency of the blue laser with another heated vapor cell. The ECDL and blue laser fields counter-propagated in the measurement. We were able to lock the sum of the two laser frequencies to the EIT transition with the root-mean-square fluctuation of around $150 \mathrm{kHz}^{47}$. 
Determination of experimental parameters. The parameters of the OD $(\alpha)$, coupling Rabi frequency $\left(\Omega_{c}\right)$, and intrinsic decoherence rate $\left(\gamma_{0}\right)$ were determined experimentally with the same method used in Ref. ${ }^{47}$. Details of the determination procedure can be found in Supplementary Note 2. We set $\Omega_{c}=1.0 \Gamma$ and were able to maintain $\gamma_{0}$ around $0.012(1) \Gamma$ throughout the experiment. This $\gamma_{0}$ included the effects of laser frequency fluctuation, Doppler shift, and other decoherence processes that appear in the $\Lambda$-type EIT system ${ }^{47}$.

Beat-note interferometer. We employed the beat-note interferometer to measure the phase shift of the probe field induced by the atoms. The concept and illustration of the beat-note interferometer can be found in Refs. ${ }^{60,61}$. In the absence of the DDI, the phase shift of the probe field also enabled us to precisely determine the probe frequency for the zero two-photon detunings, i.e., $\delta=0$, at a given coupling detuning, $\Delta_{c}$. This is because the phase shift is equal to $\tau_{d} \delta$ according to the non-DDI EIT theory, where $\tau_{d}$ is the propagation delay time. The data of attenuation coefficients and phase shifts under the DDI effect presented in Fig. 2 were taken at $\delta=0$. See Supplementary Note 1 for more details.

\section{Data availability}

The data and information within this paper are available from the corresponding author upon request.

Received: 30 October 2020; Accepted: 15 April 2021;

Published online: 20 May 2021

\section{References}

1. Lukin, M. D. et al. Dipole blockade and quantum information processing in mesoscopic atomic ensembles. Phys. Rev. Lett. 87, 037901 (2001).

2. Tong, D. et al. Local blockade of Rydberg excitation in an ultracold gas. Phys. Rev. Lett. 93, 063001 (2004).

3. Heidemann, R. et al. Evidence for coherent collective Rydberg excitation in the strong blockade regime. Phys. Rev. Lett. 99, 163601 (2007)

4. Saffman, M. et al. Quantum information with Rydberg atoms. Rev. Mod. Phys. 82, 2313-2363 (2010).

5. Weimer, H. et al. A Rydberg quantum simulator. Nat. Phys. 6, 382-388 (2010).

6. Levine, $\mathrm{H}$. et al. High-fidelity control and entanglement of Rydberg-atom qubits. Phys. Rev. Lett. 121, 123603 (2018).

7. Kim, H. Detailed balance of thermalization dynamics in rydberg-atom quantum simulators. Phys. Rev. Lett. 120, 180502 (2018).

8. Wilk, T. et al. Entanglement of two individual neutral atoms using Rydberg blockade. Phys. Rev. Lett. 104, 010502 (2010).

9. Isenhower, L. et al. Demonstration of a neutral atom controlled-NOT quantum gate. Phys. Rev. Lett. 104, 010503 (2010).

10. Levine, H. et al. Parallel implementation of high fidelity multiqubit gates with neutral atoms. Phys. Rev. Lett. 123, 170503 (2019).

11. Tiarks, D. et al. A photon-photon quantum gate based on Rydberg interactions. Nat. Phys. 15, 124-126 (2019).

12. Saffman, M. \& Walker, T. G. Creating single-atom and single-photon sources from entangled atomic ensembles. Phys. Rev. A 66, 065403 (2002).

13. Li, L. \& Kuzmich, A. Quantum memory with strong and controllable Rydberg-level interactions. Nat. Commun. 7, 13618 (2016).

14. Ripka, F. et al. A room-temperature single-photon source based on strongly interacting Rydberg atoms. Science 362, 446-449 (2018).

15. Fleischhauer, M. et al. Electromagnetically induced transparency: optics in coherent media. Rev. Mod. Phys. 77, 633-673 (2005).

16. Chen, Y.-F. et al. Low-light-level cross-phase-modulation based on stored light pulses. Phys. Rev. Lett. 96, 043603 (2006).

17. Chen, Y.-H. et al. Demonstration of the interaction between two stopped light pulses. Phys. Rev. Lett. 108, 173603 (2012).

18. Chen, Y.-H. et al. Coherent optical memory with high storage efficiency and large fractional delay. Phys. Rev. Lett. 110, 083601 (2013).

19. Hsiao, Y.-F. et al. Highly efficient coherent optical memory based on electromagnetically induced transparency. Phys. Rev. Lett. 120, 183602 (2018).

20. Pritchard, J. D. et al. Cooperative atom-light interaction in a blockaded Rydberg ensemble. Phys. Rev. Lett. 105, 193603 (2010).

21. Peyronel, T. et al. Quantum nonlinear optics with single photons enabled by strongly interacting atoms. Nature 488, 57-60 (2012).

22. Tiarks, D. et al. Optical $\pi$ phase shift created with a single-photon pulse. Sci. Adv. 2, e1600036 (2016)

23. Petrosyan, D. et al. Electromagnetically induced transparency with Rydberg atoms. Phys. Rev. Lett. 107, 213601 (2011).

24. Firstenberg, O. et al. Nonlinear quantum optics mediated by Rydberg interactions. J. Phys. B 49, 152003 (2016).

25. Moos, M. et al. Many-body physics of Rydberg dark-state polaritons in the strongly interacting regime. Phys. Rev. A 92, 053846 (2015).
26. Juzeliūnas, G. \& Carmichael, H. J. Systematic formulation of slow polaritons in atomic gases. Phys. Rev. A 65, 021601(R) (2002)

27. Fleischhauer, M. \& Lukin, M. D. Quantum memory for photons: dark-state polaritons. Phys. Rev. A 65, 022314 (2002).

28. Fleischhauer, M. et al. Bose-Einstein condensation of stationary-light polaritons. Phys. Rev. Lett. 101, 163601 (2008).

29. Fleischhauer, M. \& Lukin, M. D. Dark-state polaritons in electromagnetically induced transparency. Phys. Rev. Lett. 84, 5094-5097 (2000).

30. Anderson, M. H. et al. Observation of Bose-Einstein condensation in a dilute atomic vapor. Science 269, 198-201 (1995).

31. Davis, K. B. et al. Bose-Einstein condensation in a gas of sodium atoms. Phys Rev. Lett. 75, 3969-3973 (1995).

32. Demokritov, S. O. et al. Bose-Einstein condensation of quasi-equilibrium magnons at room temperature under pumping. Nature 443, 430-433 (2006)

33. Kasprzak, J. et al. Bose-Einstein condensation of exciton polaritons. Nature 443, 409-414 (2006).

34. Balili, R. et al. Bose-Einstein condensation of microcavity polaritons in a trap. Science 316, 1007-1010 (2007).

35. Klaers, J. et al. Bose-Einstein condensation of photons in an optical microcavity. Nature 468, 545-548 (2010).

36. Deng, H. et al. Exciton-polariton Bose-Einstein condensation. Rev. Mod. Phys. 82, 1489-1537 (2010).

37. Miesner, H. J. et al. Bosonic stimulation in the formation of a Bose-Einstein condensate. Science 279, 1005-1007 (1998).

38. Otterbach, J. et al. Wigner crystallization of single photons in cold Rydberg ensembles. Phys. Rev. Lett. 111, 113001 (2013).

39. Han, J. et al. Spectral shift and dephasing of electromagnetically induced transparency in an interacting Rydberg gas. Phys. Rev. A 94, 043806 (2016).

40. Raitzsch, U. et al. Investigation of dephasing rates in an interacting Rydberg gas. New J. Phys. 11, 055014 (2009).

41. Firstenberg, O. et al. Attractive photons in a quantum nonlinear medium. Nature 502, 71-75 (2013).

42. Jiao, Y.-C. et al. Electromagnetically induced transparency in a cold gas with strong atomic interactions. Chin. Phys. Lett. 33, 123201 (2016).

43. Liang, Q.-Y. et al. Observation of three-photon bound states in a quantum nonlinear medium. Science 359, 783-786 (2018).

44. Sinclair, J. et al. Observation of a large, resonant, cross-Kerr nonlinearity in a cold Rydberg gas. Phys. Rev. Res. 1, 033193 (2019).

45. Klaers, J. et al. Thermalization of a two-dimensional photonic gas in a 'white wall' photon box. Nat. Phys. 6, 512-515 (2010).

46. Rodriguez, S. R. K. et al. Thermalization and cooling of plasmon-exciton polaritons: towards quantum condensation. Phys. Rev. Lett. 111, 166802 (2013).

47. Kim, B. et al. Effect of laser-frequency fluctuation on the decay rate of Rydberg coherence. Phys. Rev. A 100, 013815 (2019).

48. Lin, Y.-W. et al. Using a pair of rectangular coils in the MOT for the production of cold atom clouds with large optical density. Opt. Express 16, 3753-3761 (2008)

49. Lee, M.-J. et al. Experimental demonstration of spinor slow light. Nat. Commun. 5, 5542 (2014).

50. Beterov, I. I. et al. Quasiclassical calculations of blackbody-radiation-induced depopulation rates and effective lifetimes of Rydberg $n S, n P$, and $n D$ alkalimetal atoms with $n \leq 80$ (Phys. Rev. A79, 052504 (2009); Erratum). Phys. Rev. A 80, 059902(E) (2009).

51. Walker, T. G. \& Saffman, M. Consequences of Zeeman degeneracy for the van der Waals blockade between Rydberg atoms. Phys. Rev. A 77, 032723 (2008).

52. Weatherill, K. J. et al. Electromagnetically induced transparency of an interacting cold Rydberg ensemble. J. Phys. B 41, 201002 (2008).

53. Tresp, C. et al. Dipolar dephasing of Rydberg D-state polaritons. Phys. Rev. Lett. 115, 083602 (2015).

54. Hsiao, S.-S. et al. Mean field theory of weakly-interacting Rydberg polaritons in the EIT system based on the nearest-neighbor distribution. Opt. Express 28 28414-28429 (2020)

55. Chandrasekhar, S. Stochastic problems in physics and astronomy. Rev. Mod. Phys. 15, 1-89 (1943).

56. Sevinçli, S. et al. Nonlocal nonlinear optics in cold Rydberg gases. Phys. Rev. Lett. 107, 153001 (2011).

57. Robert-de-Saint-Vincent, M. et al. Spontaneous avalanche ionization of a strongly blockaded Rydberg gas. Phys. Rev. Lett. 110, 045004 (2013).

58. Moseley, R. R. et al. Electromagnetically-induced focusing. Phys. Rev. A 53, 408-415 (1996).

59. Ravets, S. et al. Measurement of the angular dependence of the dipole-dipole interaction between two individual Rydberg atoms at a Förster resonance. Phys. Rev. A 92, 020701(R) (2015).

60. Chen, Y.-F. et al. Beat-note interferometer for direct phase measurement of photonic information. Phys. Rev. A 72, 033812 (2005).

61. Chen, Y.-H. et al. Fidelity of electromagnetically-induced-transparency-based optical memory. Phys. Rev. A 88, 023805 (2013). 


\section{Acknowledgements}

This work was supported by Grant Nos. 105-2923-M-007-002-MY3, 108-2639-M-007001-ASP, and 109-2639-M-007-002 of the Ministry of Science and Technology of Taiwan, Project No. TAP LLT-2/2016 of the Research Council of Lithuania, and Project No. LV-LT-TW/2018/7 of the Ministry of Education and Science of Latvia. JR and GJ also acknowledge support from the National Center for Theoretical Sciences, Taiwan.

\section{Author contributions}

I.A.Y. conceived the idea of a weakly-interacting many-body system of Rydberg polaritons. S.-S.H., G.J., J.R., T.K., M.A., and I.A.Y. developed the theoretical foundation for the idea. B.K., K.-T.C., Y.-C.C., Y.-F.C., and I.A.Y. designed the experimental setup and methods. B.K., K.-T.C., S.-Y.W., and K.-B.L. built the setup, carried out the experiment, and analyzed the data. The manuscript was written by B.K., S.-S.H., and I.A.Y. with help from all the other authors.

\section{Competing interests}

The authors declare no competing interests.

\section{Additional information}

Supplementary information The online version contains supplementary material available at https://doi.org/10.1038/s42005-021-00604-5.
Correspondence and requests for materials should be addressed to I.A.Y.

Reprints and permission information is available at http://www.nature.com/reprints

Publisher's note Springer Nature remains neutral with regard to jurisdictional claims in published maps and institutional affiliations.

cc (i) Open Access This article is licensed under a Creative Commons Attribution 4.0 International License, which permits use, sharing, adaptation, distribution and reproduction in any medium or format, as long as you give appropriate credit to the original author(s) and the source, provide a link to the Creative Commons license, and indicate if changes were made. The images or other third party material in this article are included in the article's Creative Commons license, unless indicated otherwise in a credit line to the material. If material is not included in the article's Creative Commons license and your intended use is not permitted by statutory regulation or exceeds the permitted use, you will need to obtain permission directly from the copyright holder. To view a copy of this license, visit http://creativecommons.org/ licenses/by/4.0/

(C) The Author(s) 2021 